\title{
Clinical Complexity in Medicine: A Measurement Model of Task and Patient Complexity
}

\author{
R. Islam'; C. Weir'; G. Del Fiol ${ }^{2}$ \\ ${ }^{1}$ University of Utah, Biomedical Informatics, School of Medicine, Salt Lake City, Utah, USA; \\ ${ }^{2}$ University of Utah, Biomedical Informatics, Salt Lake City, Utah, USA
}

\begin{abstract}
Keywords
Decision Complexity, Decision Support Systems, Clinical/Utilization, Social Medicine/ Methods, Humans, Quality Assurance, Health Care/methods, Software Design, Information Technology
\end{abstract}

\section{Summary}

Background: Complexity in medicine needs to be reduced to simple components in a way that is comprehensible to researchers and clinicians. Few studies in the current literature propose a measurement model that addresses both task and patient complexity in medicine.

Objective: The objective of this paper is to develop an integrated approach to understand and measure clinical complexity by incorporating both task and patient complexity components focusing on the infectious disease domain. The measurement model was adapted and modified for the healthcare domain.

Methods: Three clinical infectious disease teams were observed, audio-recorded and transcribed. Each team included an infectious diseases expert, one infectious diseases fel-

Correspondence to:

Roosan Islam, PharmD

University of Utah

Department of Biomedical Informatics

421 Wakara Way, Ste 140

Salt Lake City, UT 84108-3514

USA

E-mail: roosan.islam@utah.edu low, one physician assistant and one pharmacy resident fellow. The transcripts were parsed and the authors independently coded complexity attributes. This baseline measurement model of clinical complexity was modified in an initial set of coding processes and further validated in a consensus-based iterative process that included several meetings and email discussions by three clinical experts from diverse backgrounds from the Department of Biomedical Informatics at the University of Utah. Inter-rater reliability was calculated using Cohen's kappa.

Results: The proposed clinical complexity model consists of two separate components. The first is a clinical task complexity model with 13 clinical complexity-contributing factors and 7 dimensions. The second is the patient complexity model with 11 complexitycontributing factors and 5 dimensions.

Conclusion: The measurement model for complexity encompassing both task and patient complexity will be a valuable resource for future researchers and industry to measure and understand complexity in healthcare.

Methods Inf Med 2016; 55: 14-22

http://dx.doi.org/10.3414/ME15-01-0031

received: March 1, 2015

accepted: June 25, 2015

epub ahead of print: September 25, 2015

\section{Introduction}

The degree of complexity involved in medical decision-making has been increasing exponentially and has been a topic of interest for the last several decades [1-9]. With each new clinical discovery, the complexity of diagnostic, therapeutic and preventive decision-making increases. The advent of genomic medicine and the explosion of translational data are making clinical decisionrelated tasks more complex and dynamic $[10,11]$. Fields such as cybernetics, general systems theory, chaos theory, game theory, artificial life and some aspects of artificial intelligence provide a good theoretical background for designing methods to measure complexity, but may not be directly translatable to medical decision-making. Being able to model complexity in medical contexts would be useful for many purposes, including decision-support design, workflow modeling and communication interventions. Many fields have found that using models to reduce complexity helps clarify the domain cognitively $[5,7]$.

Previous studies focused on patient factors that contribute to complexity $[12,13]$. For example, the concepts of multi-morbidity, psychosocial factors and frailty have helped our understanding by reducing patient complexity to specific dimensions. These factors are mostly derived from the subjective experience of the providers or from the literature review. However, measuring and reducing complex decisions to its objective properties have not been studied as extensively in medicine as it has in other fields [14-20]. In this study, we adapted two models of complexity from other successful fields such as aviation and military to form the basis for a new, more integrated and targeted taxonomy that can be generalized in medicine. We are integrating the two perspectives, patient and task complexity.

Liu et al. have successfully conceptualized the theoretical foundation for a task complexity model from different fields and have provided a clear-cut and indepth taxonomy of decision task complex- 
ity [21]. Schaink et al. have addressed the medical domain and have done research to create a simplified model of patient complexity [22]. The Schaink model also captures the vector models of patient complexity from Safford et al. [23]. However, this model has not been validated. Both models were synthesized from a description of the objective properties of decision task and patient complexity from a review of the literature. However, although the task complexity framework has been developed by a careful study of many different domains including aviation, the military, nuclear power plants, etc., it did not include healthcare. As a result, some of the domains identified in this framework might not be congruent with the medical domain. Therefore, to address this gap, we propose to adapt the measurement models of Schaink et al. and Liu et al. as a general initial framework of clinical complexity and to identify and validate the relevant complexity-contributing factors and dimensions within the context of healthcare using human judgment.

Although our assumption is that the proposed model may help to understand the complexity factors in different domains of medicine, we are specifically focusing on the infectious disease (ID) domain because the interplay among the disease (which is often changing), the patient's response (which is not always predictable) and population-based issues of immunity and resistance often results in difficult cases [24-26]. Future electronic health records need to be designed to deal effectively with emerging infections and population health data [27-30]. Therefore, we have used the infectious disease domain for validating our proposed model.

\section{Methods}

\subsection{Settings}

The settings were two tertiary care hospitals in the United States: the University of Utah Hospital and the Salt Lake City Veterans Affairs (VA) Hospital. The University of Utah Institutional Review Board approved the study and all participants consented with a verbal waiver.

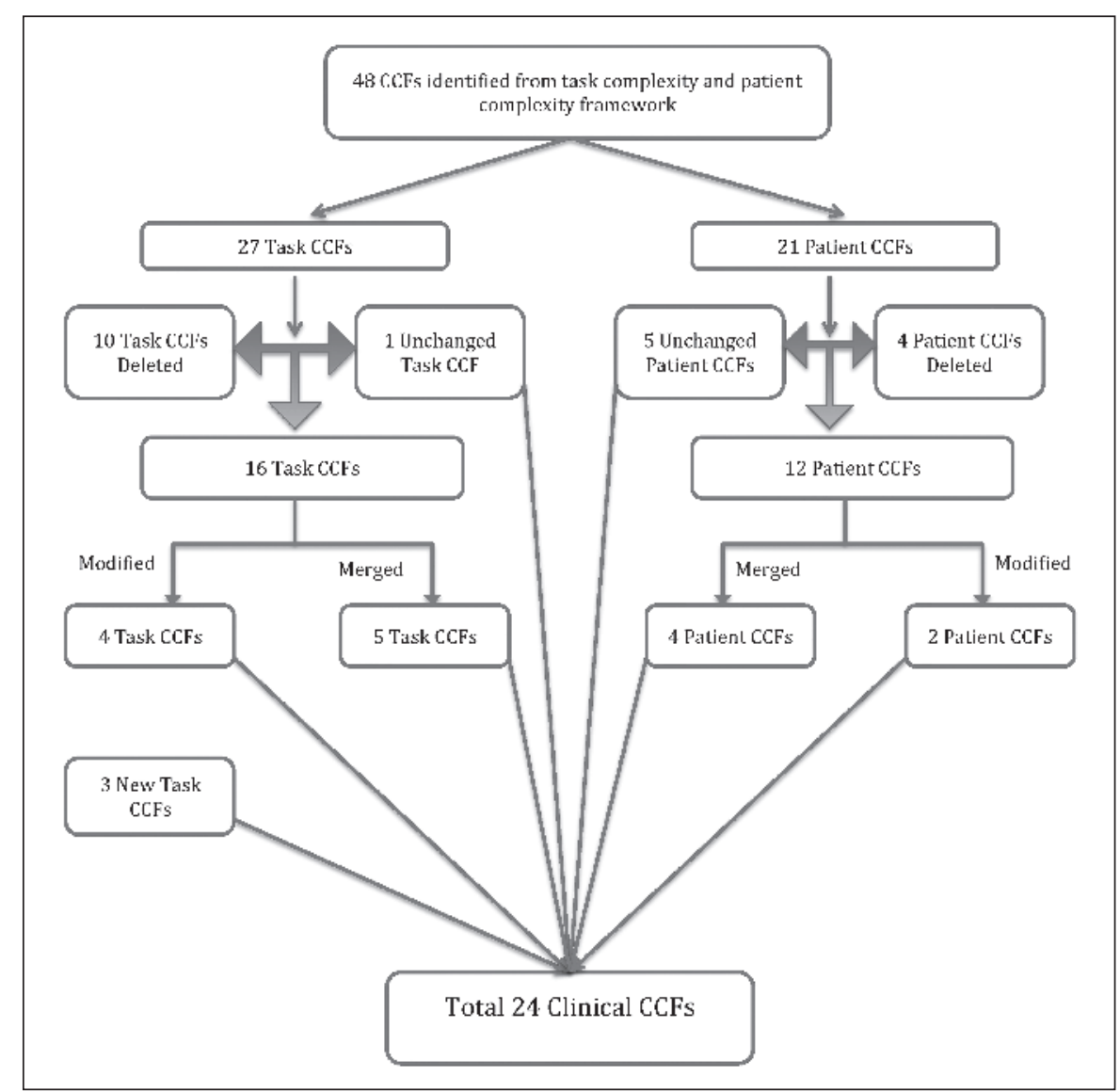

Figure 1 Complexity-contributing factors (CCFs) selection process

\subsection{Description of Observations}

Observations were conducted with the inpatient infectious disease house staff teams. Our sample size for the observation study was 30 cases. Previous studies have suc- cessfully used cases ranging from 16 to 30 [31-33]. Each case observation lasted four days from the initial consultation handed to the ID team. Each clinical team consisted of an ID expert, one ID fellow, a physician's assistant and one pharmacy
Table 1 Examples of de-identified unitized texts and associated codes

\begin{tabular}{|c|c|}
\hline Unitized texts & Associated Codes \\
\hline $\begin{array}{l}\text { It can cause a purulent infection so I don't know. These were cultures that } \\
\text { were done and everyone has got coag-negative Staph. So, I don't know if } \\
\text { that even counts for this. We just don't have any culture results. }\end{array}$ & Lack of Expertise \\
\hline $\begin{array}{l}\text { So I think actually, if you were to follow the guidelines in him, I don't } \\
\text { think Vancomycin is usually a go-to medication, it might be Unasyn. But } \\
\text { we will start both medications. }\end{array}$ & Decision Conflict \\
\hline $\begin{array}{l}\text { There are other options as well. For example, Ciprofloxacin or clindamycin. } \\
\text { I think it is fine for right now. }\end{array}$ & $\begin{array}{l}\text { Multiple Decision } \\
\text { Making Options }\end{array}$ \\
\hline $\begin{array}{l}\text { The guy is telling me that his toe is worse now on Unasyn and Vancomy- } \\
\text { cin. So, it would be nice to get better gram- negative coverage but really } \\
\text { what I think the question is if there is a little fluid collection in there or not. }\end{array}$ & Confusing Information \\
\hline $\begin{array}{l}\text { But the toe is getting worse and that is more what I would be worried } \\
\text { about. I don't know if it is from his bruising it or not. }\end{array}$ & Changing Information \\
\hline
\end{tabular}


resident. Daily rounds for the entire team were recorded and transcribed. All transcripts were de-identified and then analyzed for developing and validating the measurement model.

\subsection{Description of Reviewers}

The three authors conducted the analysis. All three are researchers and represent the diverse healthcare backgrounds of nursing,

pharmacy and medicine. Each researcher has more than five years of clinical experience and an extensive research background in healthcare and informatics, especially in clinical decision-making.

\subsection{Procedures}

The measurement model was developed by a standardized process to represent and maximize the content domain ac-

Table 2 All candidate task and patient complexity contributing factors

\begin{tabular}{|c|c|c|c|}
\hline \multicolumn{2}{|c|}{ Task complexity } & \multicolumn{2}{|c|}{ Patient complexity } \\
\hline Dimensions & Complexity Factors & Dimensions & Complexity Factors \\
\hline \multirow[t]{5}{*}{ Goal/Output } & Clarity & \multirow{4}{*}{$\begin{array}{l}\text { Medical/physi- } \\
\text { cal health }\end{array}$} & Loss of physical functioning \\
\hline & Quantity & & Polypharmacy \\
\hline & Conflict & & $\begin{array}{l}\text { Limited application of clinical } \\
\text { practice guidelines }\end{array}$ \\
\hline & Redundancy & & Multimorbidity \\
\hline & Change & \multirow[t]{4}{*}{ Mental health } & Psychological distress \\
\hline \multirow[t]{10}{*}{ Input } & Clarity & & Psychiatric illness \\
\hline & Quantity & & Cognitive impairment \\
\hline & Diversity & & Addictions/substance abuse \\
\hline & Inaccuracy & \multirow[t]{5}{*}{ Demographics } & Older age \\
\hline & Rate of change & & Frailty \\
\hline & Redundancy & & Female gender \\
\hline & Conflict & & Ethnic disparities \\
\hline & Unstructured guidance & & Lower education \\
\hline & Mismatch & \multirow[t]{4}{*}{ Social capital } & Negatively affected relationships \\
\hline & Non-routine events & & Caregiver strain and burnout \\
\hline \multirow[t]{7}{*}{ Process } & Clarity & & $\begin{array}{l}\text { Low socio-economic status and } \\
\text { poverty }\end{array}$ \\
\hline & Quantity of paths & & Poor social support \\
\hline & Quantity of actions/steps & \multirow{5}{*}{$\begin{array}{l}\text { Health and } \\
\text { social experi- } \\
\text { ences }\end{array}$} & $\begin{array}{l}\text { Heavy utilization of healthcare } \\
\text { resources }\end{array}$ \\
\hline & Conflict & & Costly care \\
\hline & Repetitiveness & & Self-management challenges \\
\hline & $\begin{array}{l}\text { Cognitive requirements by an } \\
\text { action }\end{array}$ & & Poor quality of life \\
\hline & $\begin{array}{l}\text { Physical requirement by an } \\
\text { action }\end{array}$ & & $\begin{array}{l}\text { Difficulty with healthcare system } \\
\text { navigation }\end{array}$ \\
\hline \multirow[t]{2}{*}{ Time } & Concurrency & & \\
\hline & Pressure & & \\
\hline \multirow[t]{3}{*}{ Presentation } & Format & & \\
\hline & Heterogeneity & & \\
\hline & Compatibility & & \\
\hline
\end{tabular}

cording to Lynn's recommendation [34]. The procedure for developing and validating the measurement model included five steps:

1) Descriptions of initial model revisions,

2) unitizing texts from interview transcripts,

3) expert panel content coding for validation,

4) modification of categories through discussion and assessment of reliability and

5) iterative recoding and modification of categories.

This overall process is described in $>$ Figure 1.

\subsubsection{Data Analysis}

The data analysis was based on content analysis [35]. Specifically, we have followed the "emergent coding" process of content analysis [36]. In this process, researchers independently review a subset of the data and form a checklist for coding. After independently coding, they meet to discuss and reconcile the differences. Once the coding has reached the desired reliability, then it is applied to the remainder of the data.

Also, we have used the RATS (Relevance of study question, Appropriateness of qualitative method, Transparency of procedure and Soundness of interpretive approach) protocol for qualitative data analysis for the transcriptions of the interviews [37]. This protocol provides standardized guidelines for qualitative research methods.

\subsubsection{Description of Initial Model Revisions}

A list of 49 candidate complexity-contributing factors (CCFs) was adapted from the task and patient complexity review by Liu et al. and Schaink et al. [21, 22]. From those, 27 task-related CCFs were identified. Factors not relevant to medical care were removed. In addition, 22 CCFs from the patient complexity perspective were identified. The 49 total CCFs identified from the initial models served as the coding framework for the transcripts from the observation study. 


\subsubsection{Unitizing Texts from Interview Transcripts}

One researcher unitized or parsed the texts to prepare for coding. Each unit consisted of one or more sentences that conveyed one idea. Although content can be unitized in multiple ways, the three investigators reviewed and agreed with the units during the coding process. Fifty unitized sections were used for each iteration. We used the ATLAS.ti-7.5 qualitative data analysis software package for unitizing the texts, text segmentations, attaching the codes to the segments, merging and combining codes and for coding and retrieval strategies that facilitated forming the final codes and the connections among the codes. The other two researchers reviewed the unitized segments for consistency and accuracy. In - Table 1, we provide some de-identified unitized texts and the associated codes.

\subsubsection{Expert Panel (EP) Content Coding for Validation}

One researcher unitized the texts and the other two researchers independently coded each unitized text based on the 49 CCFs. In

- Table 2, we have included all the initial candidate factors.

\subsubsection{Modification of Categories through Discussion and Assessment of Reliability}

After each coding session, the three researchers met to examine coding disagreements and to revise codes and code definitions. Cohen's kappa was calculated after each revision. The final inter-rater reliability of Cohen's kappa was 0.8 .

\subsubsection{Iterative Recoding and Modification of Categories}

As a result of discussion, codes were merged, deleted and renamed. This process was repeated four times. For each iteration, the expert panel validated the codes by matching the unitized text with one and only one code. When a text could not be coded, a new category was created and then retested across additional text units.

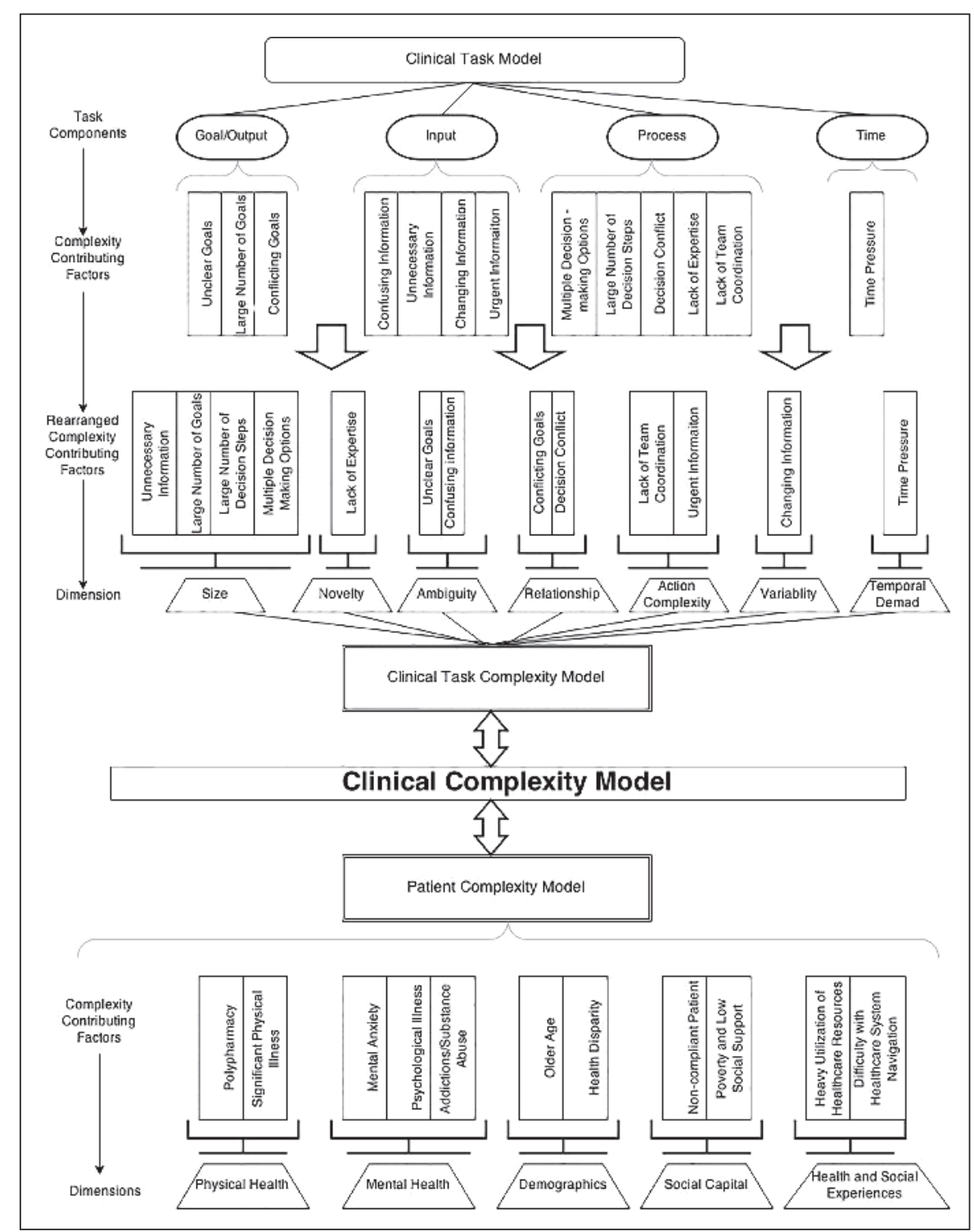

Figure 2 Clinical Complexity Model

\section{Results}

The results are organized into two sections. In the first section, the formation of the clinical task and patient CCFs is described. In the second section, we integrated the CCFs into higher-order dimensions. The conceptualized framework for clinical complexity is shown in $>$ Figure 2.

\subsection{Clinical Task and Patient Complexity-contributing Factors}

Overall, out of the 49 CCFs, 13 task CCFs and 11 patient CCFs were identified as rel- evant to healthcare. Detailed descriptions of each CCF are in $>$ Table 3.

A total of 6 CCFs ( 5 patient CCFs and 1 task $\mathrm{CCF}$ ) remained unchanged from the initial 49 CCFs including polypharmacy, addictions/substance abuse, older age, heavy utilization of healthcare resources, difficulty with healthcare system navigation and time pressure.

The selection of the CCFs consisted of three types of activities:

i) relevant items modified,

ii) items removed as not relevant and

iii) new items generated. The overall process is described in $\mathbf{\nabla}$ Figure 3. 


\begin{tabular}{|c|c|c|}
\hline & CCFs & Definitions \\
\hline \multirow{13}{*}{$\begin{array}{l}\text { Task complexity } \\
\text { contributing } \\
\text { factors }\end{array}$} & Unclear goals & Objective is unclear or vague, less clear or specific goals \\
\hline & Large number of goals & Multiple goal elements, higher or larger number of goals \\
\hline & Conflicting goals & Achieving one goal has negative effect or outcome on another goal \\
\hline & Confusing information & Unclear, missing, ambiguous or contradictory information cues \\
\hline & Unnecessary information & Large quantity of not useful information \\
\hline & Changing information & Unpredictable events, high rate of information change \\
\hline & Urgent information & Information about very acute patient situation \\
\hline & Multiple decision-making options & Large number of options to make a decision \\
\hline & Large number of decision steps & More than two steps or actions to attain the objective \\
\hline & Decision conflict & $\begin{array}{l}\text { Two or more actions that are incompatible or competing, conflict } \\
\text { between task components }\end{array}$ \\
\hline & Lack of expertise & $\begin{array}{l}\text { Unique situation requiring additional knowledge, novel and } \\
\text { non-routine decisions, treatment or disease uncertainty }\end{array}$ \\
\hline & Lack of team coordination & $\begin{array}{l}\text { Coordinating activities and creating shared decision-making within } \\
\text { and between healthcare teams }\end{array}$ \\
\hline & Time pressure & Situations that need immediate attention due to scarcity of time \\
\hline \multirow{11}{*}{$\begin{array}{l}\text { Patient complex- } \\
\text { ity contributing } \\
\text { factors }\end{array}$} & Polypharmacy & Patient receiving medications from more than one pharmacy \\
\hline & Significant physical illness & Multiple chronic conditions, loss of physical functioning \\
\hline & Mental anxiety & External factors creating cognitive stress (e.g., job, culture, family) \\
\hline & Psychological illness & Depression, mood disorders, loosing self-consciousness \\
\hline & Addiction/substance abuse & Drug or substance abuse in the past or present \\
\hline & Older age & Patient age 75 and older \\
\hline & Health disparity & $\begin{array}{l}\text { Patients with different ethnic background and cultural barrier with } \\
\text { limited access to healthcare }\end{array}$ \\
\hline & Non-compliant patient & $\begin{array}{l}\text { Patient not following medication or treatment regimen, difficulty } \\
\text { communicating with providers }\end{array}$ \\
\hline & Poverty and low social support & $\begin{array}{l}\text { Poor social support, low quality of life due to economic strains and } \\
\text { lower social status }\end{array}$ \\
\hline & $\begin{array}{l}\text { Heavy utilization of healthcare } \\
\text { resources }\end{array}$ & $\begin{array}{l}\text { Complex chronic patients with multiple care providers and institu- } \\
\text { tions require more resources }\end{array}$ \\
\hline & $\begin{array}{l}\text { Difficulty with healthcare system } \\
\text { navigation }\end{array}$ & Low understanding of healthcare system, limited healthcare literacy \\
\hline
\end{tabular}

Table 3

Clinical complexitycontributing factors (CCFs) and specific definitions

\subsubsection{Relevant Items Modified}

Overall, the EP modified and merged 16 task CCFs into 9 task CCFs. The goal clarity and goal change $\mathrm{CCF}$ were merged into unclear goals. The EP merged input conflict, clarity and inaccuracy into a general category called confusing information. Also, the input nonroutine information and input rate of change were merged into one category, called changing information. Input quantity and input diversity were merged into a new category, called unnecessary information. Process clarity, process conflict and process cognitive requirement by an action were merged into decision conflict. Process quantity of paths and process quantity of action/steps were respectively renamed multiple decision-making options and large number of decision steps.

The EP also modified and merged the 13 patient CCFs into a final set of 6 patient CCFs.

Loss of physical function leading to chronic disease, multimorbidity and frailty were merged into significant physical illness. Cognitive impairment was merged into the definition of psychological illness. Psychological distress and negative affected relation- ship were modified, respectively, to mental anxiety and non-compliant patient. Ethnic disparity and lower education were merged into a broader definition of health disparity. Then, caregiver strain and burnout, low socio-economic status and poverty, poor social support and poor quality of life were merged into poverty and low social support.

\subsubsection{Items Removed as Not Relevant}

Overall, a total of 14 complexity-contributing factors including both task (10 CCFs) 
and patient (4 CCFs) complexity-contributing factors were not used for coding the transcripts and were removed: Goal redundancy, input unstructured guidance, input mismatch, input redundancy, process repetitiveness, process physical requirement by an action, task concurrency, presentation format, presentation heterogeneity, presentation compatibility, limited clinical guidelines, female gender, costly care and self-management challenges.

\subsubsection{New Items Generated}

Overall, three new task CCFs were added: urgent information, lack of expertise and lack of team coordination.

\subsection{The Formation of Dimensions from Complexity-contributing Factors (CCFs)}

Seven clinical task complexity dimensions were grouped together from the 13 clinical task CCFs. Then, the 11 patient CCFs were grouped into 5 patient complexity dimensions. Table 4 includes a short description of the clinical task complexity and patient complexity dimensions and the criteria we used to group them. We have adapted the dimensions from the conceptualizations by Liu et al. and Schaink et al. $[21,22]$.

\section{Discussion}

In this paper, we have conceptualized and validated a clinical complexity model that includes both task complexity and patient complexity-contributing factors, and groups these factors into higher-level dimensions. To our knowledge, this is the first research that has integrated a clinical task complexity model with a patient complexity model for a better understanding of overall complexity in medicine.

Most complex patients do not fall under simple guidelines due to issues such as multi-morbidity and chronic conditions. Recent estimates indicate that more than 75 million persons in the United States have two or more concurrent chronic conditions [39]. Moreover, the aging population will contribute to in-

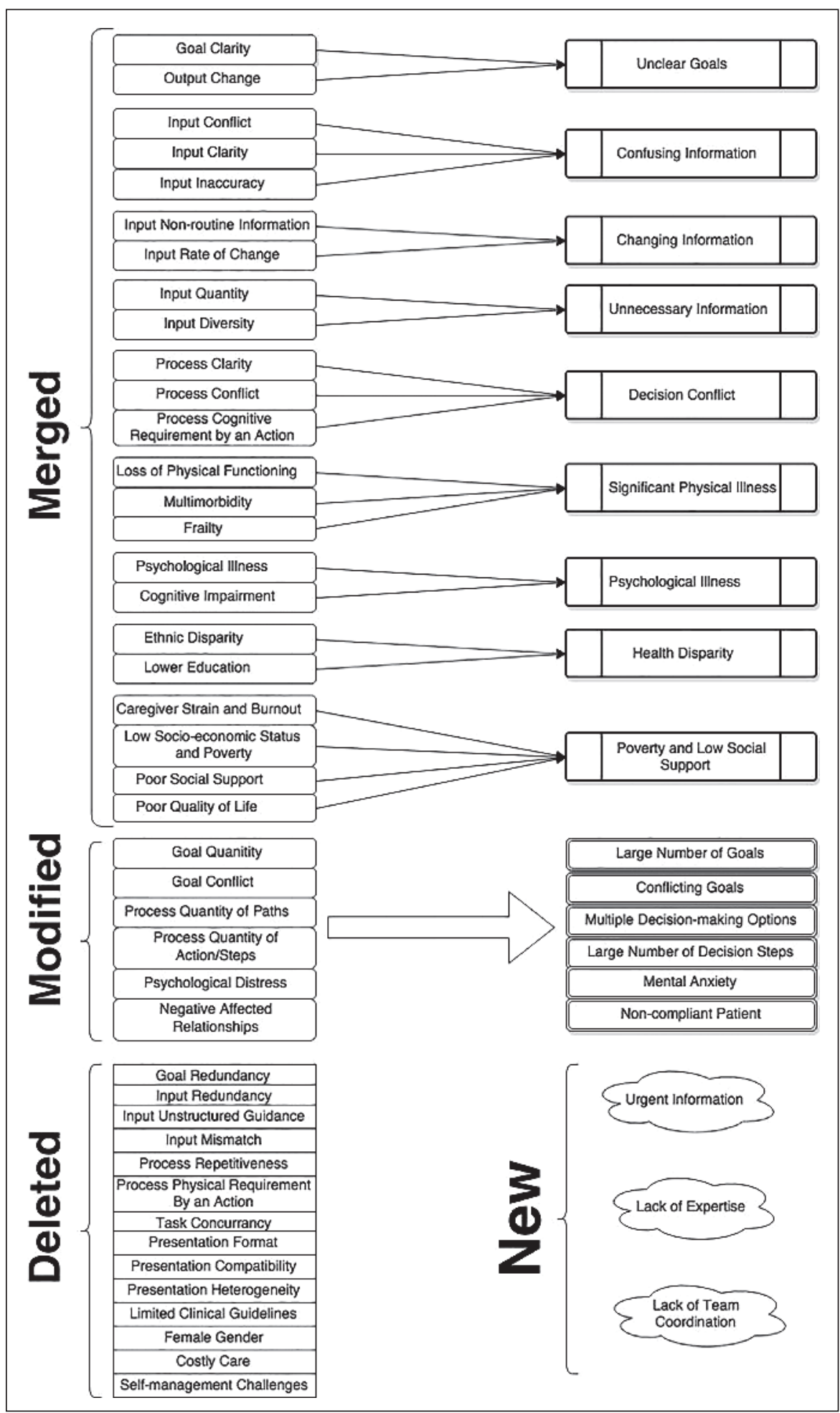

Figure 3 Overview of the merged, modified, deleted and new clinical complexity-contributing factors (CCFs)

creasing the complexity of patient presentations. Thus, managing these complex patients requires extra effort for the clinicians from both healthcare and non- healthcare resources. On the other hand, the standard quality of measures in the study population often excludes complex patients, and thus applying inappropriate 
Table 4 Dimensions, criteria and specific definitions

\begin{tabular}{|c|c|c|c|}
\hline & Dimensions & Criteria & Definition \\
\hline \multirow{7}{*}{$\begin{array}{l}\text { Clinical task } \\
\text { complexity }\end{array}$} & Size & Number, quantity & Large number of clinical tasks \\
\hline & Novelty & Uniqueness & $\begin{array}{l}\text { Novel, non-routine tasks, clinical tasks dealing with treatment or diag- } \\
\text { nostic uncertainty }\end{array}$ \\
\hline & Ambiguity & $\begin{array}{l}\text { Not clear or specific infor- } \\
\text { mation }\end{array}$ & Unclear, vague, less-specific clinical task components \\
\hline & Relationship & Connection & Incompatible, conflicting or competing clinical tasks \\
\hline & Action complexity & Shared attention & Shared cognition for task execution, acuity of the clinical task \\
\hline & Variability & Changing information & Unpredictable, high rate of changing clinical task components \\
\hline & Temporal demand & Time constraints & Clinical tasks requiring immediate action or attention due to time pressure \\
\hline \multirow[t]{5}{*}{$\begin{array}{l}\text { Patient } \\
\text { complexity }\end{array}$} & Physical health & Physical attributes & $\begin{array}{l}\text { Multimorbidity, polypharmacy, chronic conditions, loss of physical } \\
\text { functioning }\end{array}$ \\
\hline & Mental health & Psychological domains & $\begin{array}{l}\text { Psychological, cultural stress leading to mental pressure, depression, mood } \\
\text { disorders, addictions/substance abuse }\end{array}$ \\
\hline & Demographics & Background information & Age, gender ethnicities for the patient \\
\hline & Social capital & Behavior and social support & $\begin{array}{l}\text { Non-compliant patient, loss of social support, low quality of life and social } \\
\text { status }\end{array}$ \\
\hline & $\begin{array}{l}\text { Health and social experi- } \\
\text { ences }\end{array}$ & $\begin{array}{l}\text { Chronicity and impaired } \\
\text { cognition }\end{array}$ & $\begin{array}{l}\text { Complex chronic patients requiring more healthcare resources, poor under- } \\
\text { standing of overall healthcare system }\end{array}$ \\
\hline
\end{tabular}

quality measures can be a distraction for clinicians while taking care of the unmet, high-priority needs of complex patients [40-42]. As a result, clinicians have the option to select healthier patients and may reject the chronic complex patients if not properly incentivized [43]. Therefore, a model to objectively measure clinical complexity may be necessary in the coming era of pay-for-performance. The proposed model can fill that gap and objectively measure clinical complexity for the daily practice of medicine.

Moreover, complex patients lead to information overload and decision uncertainty even for expert clinicians $[1,3,13$, $44,45]$. As a result, clinicians tend to overlook important information cues, resulting in diagnostic and therapeutic errors [46-51]. Understanding the factors underlying complex clinical decisionmaking can be used to guide future electronic health record and clinical decision support designers. For example, if unclear goals are more prominent in the first few days of inpatient admissions, then decision support design should incorporate a goal-directed and task-centered approach. This approach provides a shared sense of situation awareness among team members. Thus, by adopting such an approach, system designers can help to mitigate communication errors and improve clinical workflow efficiency. Goaldirected task analysis, when incorporated into visual interface design, has been shown to improve group decision-making in other domains, such as aviation and the military [52-54]. The complexity factors that are identified for certain domains using this measurement model may help guide the design of EHR functionality to help clinicians cope with complexity.

In this study, we adopted models from non-healthcare fields and applied them to healthcare. In the process, we added new complexity contributing-factors and more specifically, the integration of task and patient complexity factors including expert review. Future studies may address this initial reference model with other reference models for comparison by using physicians' subjective judgment. Also, future studies in different clinical domains may validate whether the proposed model can adequately capture all components of complexity.

\section{Limitations}

A limitation might be the generalizability to other clinical domains. Infectious disease is a very complex and dynamic domain. Thus, the complexity it entails is likely to give a reasonable representation of the complexity in healthcare. However, other clinical domains might present some diverse and unique complexity-contributing factors. Therefore, future research can probe into other clinical domains by using our framework. Additional findings of complexity-contributing factors from different domains of medicine can help simplify complexity even further. The fact that all the investigators were involved in the coding process may have introduced some bias. However, the researchers had different clinical and scientific backgrounds that may have helped to reduce any coding biases. Also, this study was conducted in only two hospitals in Utah. Thus, it is unknown whether the results can be generalized to other settings. Nevertheless, the patients, clinicians, and study sites are typical representations of academic medical centers in the US. Another limitation of this study is that the clinical complexity 
captured in this study was limited to conversations among the ID clinical team. Other complexity factors may arise from interactions between patients and providers, between physicians and other types of providers who did not participate in the rounds, and as part of other care coordination activities.

\section{Conclusion}

This study proposes a systematic understanding of complexity in medicine. The resulting clinical complexity model consists of 24 clinical complexity-contributing factors, including both patient and task factors, organized into 12 dimensions. The model can help researchers in academia and industry to develop and evaluate healthcare systems. Also, the proposed model can be useful for system design, task design, work organization, human-system interaction, human performance and behavior, system safety and many other applications.

\section{Acknowledgment}

This project was supported by the grant number R36HS023349 from the Agency for Healthcare Research and Quality (AHRQ). This work was also supported in part by National Library of Medicine training grant T15-LM07124, and two grants from the department of Veterans Affairs (CRE 12-321 and CRE 12-230).

\section{References}

1. Bedny GZ, Karwowski W, Bedny IS. Complexity Evaluation of Computer-Based Tasks. International Journal of Human-Computer Interaction 2012; 28 (4): 236-257.

2. Braithwaite RS, Concato J. Vector Model of Complexity. J Gen Intern Med 2008; 23 (6): 895.

3. Jenkins DP. Cognitive Work Analysis: Coping with Complexity: Ashgate Publishing, Ltd.; 2009.

4. Kannampallil TG, Schauer GF, Cohen T, Patel VL. Considering Complexity in Healthcare Systems. Journal of Biomedical Informatics 2011; 44 (6): 943-947.

5. Plsek PE, Wilson T. Complexity Science: Complexity, Leadership, and Management in Healthcare Organisations. British Medical Journal 2001; 323 (7315): 746-749.

6. Sintchenko V, Coiera E. Decision Complexity Affects the Extent and Type of Decision Support Use. AMIA Annu Symp Proc 2006: 724-728.
7. Wilson T, Holt T. Complexity Science: Complexity and Clinical Care. British Medical Journal 2001; 323 (7314): 685-688.

8. Woods DD. Coping with Complexity: The Psychology of Human Behaviour in Complex Systems. Tasks, Errors and Mental Models 1988: 128-148.

9. Moura Jr LA. Complexity and the Will to Transform. Methods Inf Med 2012; 51 (3): 187-188.

10. Sandoval J, Esteller M. Cancer Epigenomics: Beyond Genomics. Current Opinion in Genetics \& Development 2012; 22 (1): 50-55.

11. Alonso F, Lara JA, Martinez L, PĖrez A, Valente JP. Generating Reference Models for Structurally Complex Data. Application to the Stabilometry Medical Domain. Methods Inf Med 2013; 52 (5): 441- 453 .

12. Grant RW, Ashburner JM, Hong CS, Chang Y, Barry MJ, Atlas SJ. Defining Patient Complexity From the Primary Care Physician's Perspective: A Cohort Study. Annals of Internal Medicine 2011; 155 (12): 797-804.

13. Shippee ND, Shah ND, May CR, Mair FS, Montori VM. Cumulative Complexity: A Functional, Patient-Centered Model of Patient Complexity Can Improve Research and Practice. J Clin Epidemiol 2012; 65 (10): 1041-1051.

14. Huntley MS Jr. Task Complexity and Serial Performance Under Steadily Increasing Input Rates. Human Factors 1972; 14 (1): 65-75.

15. Hartley AA, Anderson JW. Task Complexity and Problem-Solving Performance in Younger and Older Adults. Journals of Gerontology 1983; 38 (1): $72-77$.

16. Gardner DG. Task Complexity Effects on Nontask-Related Movements: A Test of Activation Theory. Organizational Behavior and Human Decision Processes 1990; 45 (2): 209-231.

17. Braarud PO. Subjective Task Complexity and Subjective Workload: Criterion Validity for Complex Team Tasks. International Journal of Cognitive Ergonomics 2001; 5 (3): 261-273.

18. Gill TG, Hicks RC. Task Complexity and Informing Science: A Synthesis. Informing Science 2006; 9: $1-30$.

19. Gill TG, Murphy WF. Task Complexity and Design Science. In: 9th International Conference on Education and Information Systems. Technologies and Applications (EISTA 2011), Orlando, FL, USA. 2011.

20. Ham DH, Park J, Jung W. Model-Based Identification and Use of Task Complexity Factors of Human Integrated Systems. Reliability Engineering and System Safety 2012; 100: 33-47.

21. Liu P, Li Z. Task Complexity: A Review and Conceptualization Framework. International Journal of Industrial Ergonomics 2012; 42 (6): 553-568.

22. Schaink AK, Kuluski K, Lyons RF, Fortin M, Jadad AR, Upshur R, et al. A Scoping Review and Thematic Classification of Patient Complexity: Offering a Unifying Framework. Journal of Comorbidity 2012; 2 (1): 1-9.

23. Safford MM, Allison JJ, Kiefe CI. Patient Complexity: More Than Comorbidity. The Vector Model of Complexity. Journal of General Internal Medicine 2007; 22 (3): 382-390.

24. Fauci AS, Morens DM. The Perpetual Challenge of Infectious Diseases. N Engl J Med. 2012; 366 (5): 454-461.
25. Fong IW. Challenges in Infectious Diseases: Springer; 2013.

26. Khabbaz RF, Moseley RR, Steiner RJ, Levitt AM, Bell BP. Challenges of Infectious Diseases in the USA. The Lancet 2014; 384 (9937): 53- 63.

27. Carroll LN, Au AP, Detwiler LT, Fu T-c, Painter IS, Abernethy NF. Visualization and Analytics Tools for Infectious Disease Epidemiology: A Systematic Review. Journal of Biomedical Informatics 2014; $51(0): 287-298$.

28. Morens DM, Folkers GK, Fauci AS. The Challenge of Emerging and Re-emerging Infectious Diseases. Nature 2004; 430 (6996): 242-249.

29. Tleyjeh IM, Nada H, Baddour LM. VisualDx: Decision-Support Software for the Diagnosis and Management of Dermatologic Disorders. Clinical Infectious Diseases 2006; 43 (9): 1177-1184.

30. McMullen CK, Ash JS, Sittig DF, Bunce A, Guappone K, Dykstra R, et al. Rapid Assessment of Clinical Information Systems in the Healthcare Setting. An Efficient Method for Time-Pressed Evaluation. Methods Inf Med 2011; 50 (4): 299-307.

31. Timmermans DR, Gooszen AW, Geelkerken RH, Tollenaar RA, Gooszen HG. Analysis of the Variety in Surgeons' Decision Strategies for the Management of Left Colonic Emergencies. Medical Care 1997; 35 (7): 701-713.

32. Timmermans DR, Sprij AJ, de Bel CE. The Discrepancy Between Daily Practice and the Policy of a Decision-Analytic Model: The Management of Fever of Unknown Origin. Medical Decision Making: An International Journal of the Society for Medical Decision Making 1996; 16 (4): 357-366.

33. Begum S, Ahmed MU, Funk P, Xiong N, Von Schéele B. A Case-Based Decision Support System for Individual Stress Diagnosis Using Fuzzy Similarity Matching. Computational Intelligence 2009; 25 (3): 180-195.

34. Lynn MR. Determination and Quantification of Content Validity. Nursing Research 1986; 35 (6): 382-386.

35. Stemler S. An Overview of Content Analysis. Practical Assessment, Research \& Evaluation 2001; 7 (17): 137-146.

36. Haney W, Russell M, Gulek C, Fierros E. Drawing on Education: Using Student Drawings to Promote Middle School Improvement. Schools in the Middle 1998; 7 (3): 38-43.

37. Clark J. How to Peer Review a Qualitative Manuscript. Peer Review in Health Sciences 2003; 2: 219-235.

38. Custer RL, Scarcella JA, Stewart BR. The Modified Delphi Technique-A Rotational Modification. Journal of Vocational and Technical Education 1999.

39. Wolff JL, Starfield B, Anderson G. Prevalence, Expenditures, and Complications of Multiple Chronic Conditions in the Elderly. Archives of Internal Medicine 2002; 162 (20): 2269-2276.

40. Werner RM, Asch DA. Clinical Concerns About Clinical Performance Measurement. The Annals of Family Medicine 2007; 5 (2): 159-163.

41. Hübner-Bloder G, Ammenwerth E. Key Performance Indicators to Benchmark Hospital Information Systems, A Delphi Study. Methods Inf Med 2009; 48 (6): 508-518.

42. Arnrich B, Mayora O, Bardram J, Tröster G. Pervasive Healthcare. Paving the Way for a Pervasive, 
User-Centered and Preventive Healthcare Model. Methods Inf Med 2010; 49 (1): 67-73.

43. Werner RM, Greenfield S, Fung C, Turner BJ. Measuring Quality of Care in Patients With Multiple Clinical Conditions: Summary of a Conference Conducted by the Society of General Internal Medicine. Journal of General Internal Medicine 2007; 22 (8): 1206-1211.

44. Turner BJ, Cuttler L. The Complexity of Measuring Clinical Complexity. Annals of Internal Medicine 2011; 155 (12): 851-852.

45. Islam R, Weir C, Fiol GD, editors. Heuristics in Managing Complex Clinical Decision Tasks in Experts' Decision Making. 2014 IEEE International Conference on Healthcare Informatics (ICHI); Sept 15-17, 2014.

46. Mane KK, Bizon C, Schmitt C, Owen P, Burchett B, Pietrobon R, et al. VisualDecisionLinc: A Visual
Analytics Approach for Comparative Effectiveness-Based Clinical Decision Support in Psychiatry. J Biomed Inform 2012; 45 (1): 101-106.

47. Singh H, Petersen LA, Thomas EJ. Understanding Diagnostic Errors in Medicine: A Lesson from Aviation. Quality and Safety in Health Care 2006; 15 (3): 159-164.

48. Elstein AS. Thinking About Diagnostic Thinking: A 30-Year Perspective. Advances in Health Sciences Education: Theory and Practice 2009; 14 Suppl 1: 7-18.

49. Schiff GD, Hasan O, Kim S, Abrams R, Cosby K, Lambert BL, et al. Diagnostic Error in Medicine: Analysis of 583 Physician-Reported Errors. Archives of Internal Medicine 2009; 169 (20): 1881-1887.

50. Graber ML, Kissam S, Payne VL, Meyer AN, Sorensen $\mathrm{A}$, Lenfestey $\mathrm{N}$, et al. Cognitive Interven- tions to Reduce Diagnostic Error: A Narrative Review. BMJ Quality \& Safety 2012; 21 (7): 535-557.

51. Croskerry P, Petrie DA, Reilly JB, Tait G. Deciding About Fast and Slow Decisions. Academic Medicine: Journal of the Association of American Medical Colleges 2014; 89 (2): 197-200.

52. Bailey BP, Iqbal ST. Understanding Changes in Mental Workload During Execution of Goal-Directed Tasks and Its Application for Interruption Management. ACM Transactions on ComputerHuman Interaction (TOCHI) 2008; 14 (4): 21.

53. John BE, Kieras DE. Using GOMS for User Interface Design and Evaluation: Which Technique? ACM Transactions on Computer-Human Interaction (TOCHI) 1996; 3 (4): 287-319.

54. Wagner T, Garvey A, Lesser V. Criteria-Directed Task Scheduling. International Journal of Approximate Reasoning 1998; 19 (1): 91-118.

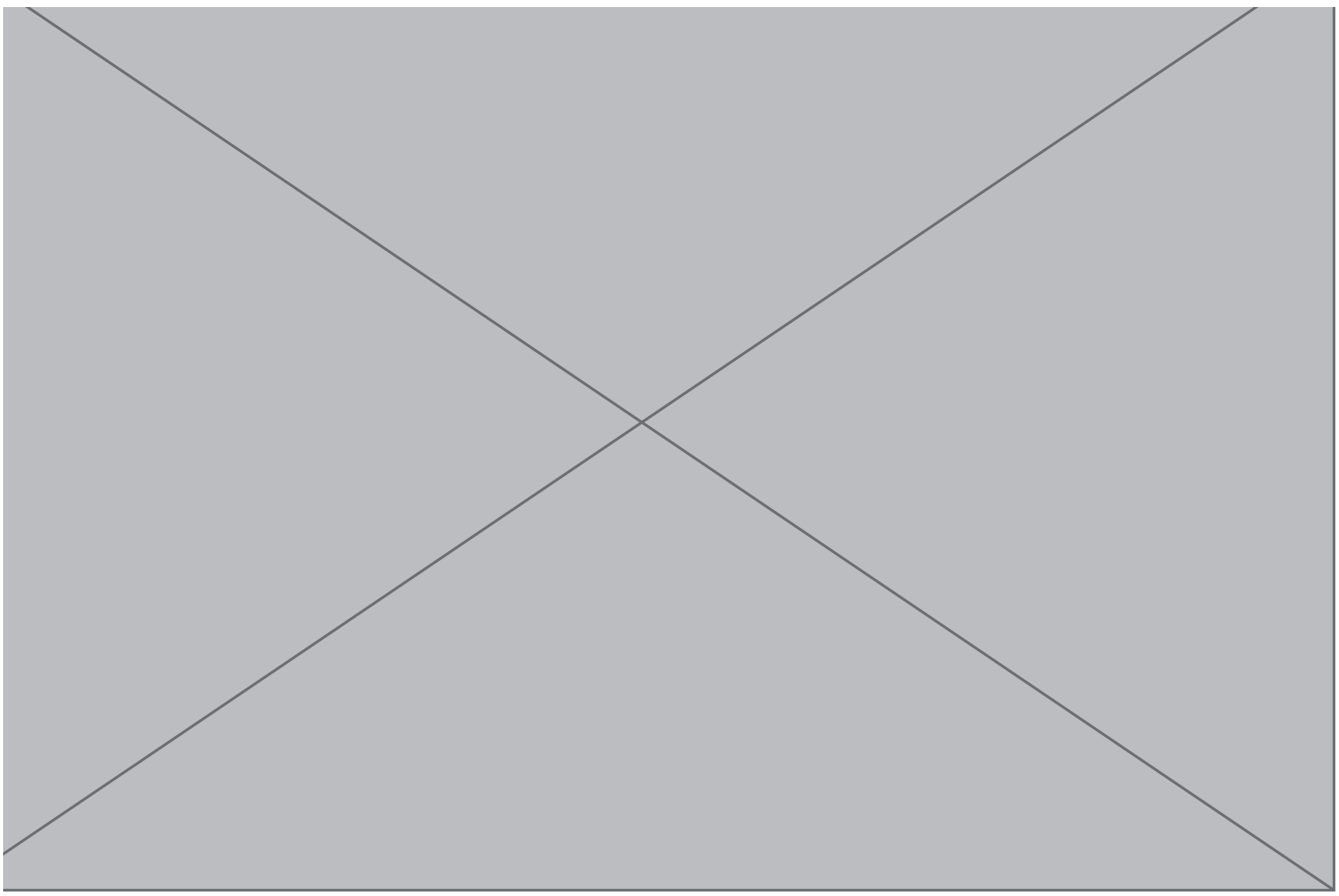

